\title{
ON TRANSFORMATIONS OF DOUBLE SERIES
}

\author{
BY H. J. HAMILTON
}

1. Introduction and Definition of Notation. A double series $\sum_{k, l=1}^{\infty} x_{k l}$ may be classified according to the behavior of the double sequence of its partial sums $s_{k l} \equiv \sum_{i, j=1}^{k, l} x_{i j}$ as follows. The sequence $\left\{s_{k l}\right\}$ is ultimately bounded (abbreviated $u b$ ) if there exists a number $Q$ such that $s_{k l}$ is bounded for all $k, l>Q$; bounded $(b)$ if in the preceding case $Q$ can be taken to be zero; convergent $(c)$ if $\lim _{k, l \rightarrow \infty} s_{k l}$ exists (finite) ; bounded convergent $(b c$ ) if both $b$ and $c$; ultimately regularly convergent (urc) if $c$, and if there exists a number $\bar{Q}$ such that $\lim _{k \rightarrow \infty} s_{k l}$ and $\lim _{l \rightarrow \infty} s_{k l}$ both exist (finite) for all $l>\bar{Q}$ and all $k>\bar{Q}$, respectively; regularly convergent $(r c)$ if in the preceding case $\bar{Q}$ can be taken to be zero; bounded ultimately regularly convergent (burc) if both $b$ and urc.

It is the purpose of the present paper to establish necessary and sufficient conditions on the matrix $\left\|b_{k l}\right\|$ in order that, whenever the series $\sum_{k, l=1}^{\infty} x_{k l}$ is of a specified one of the above types, the transformed series $\sum_{k, l=1}^{\infty} x_{k l} b_{k l}$ will be of a specified one of these types. The process of transforming will be indicated by an arrow; "sufficient" will be abbreviated by $S$., "necessary" by $N$. Thus $N . b \rightarrow c$ reads "a condition (or set of conditions) necessary that every bounded series have a convergent transform," and $S . b \rightarrow c$ reads "a condition (or set of conditions) sufficient that every bounded series have a convergent transform."

Hardy* found conditions $N$. and $S . r c \rightarrow r c$, and conditions $S . b \rightarrow r c$, and established relations (6) and (8) below. Kojima $\dagger$ proved the necessity of Hardy's conditions $S . b \rightarrow r c$, and discovered conditions $N$. and $S . c \rightarrow c$. C. N. Moore $\ddagger$ established conditions $N$. and $S . b c \rightarrow b c$ incidentally, in proving a theorem

* Hardy, On the convergence of certain multiple series, Proceedings of the Cambridge Philosophical Society, vol. 19 (1920), pp. 86-95. This paper will be referred to as $\mathrm{H}$.

$\dagger$ Kojima, Theorems on double series, Tôhoku Mathematical Journal, vol. 17 (1920), pp. 213-220. This paper will be referred to as $\mathrm{K}$.

$\ddagger$ C. N. Moore, On convergence factors in multiple series, Transactions of this Society, vol. 29 (1927), pp. 227-238. Let $r=1$, and fix $\alpha$ and $\beta$ in Moore's Theorem 1. 
on Cesàro transforms of double series; the proof of (10) below is

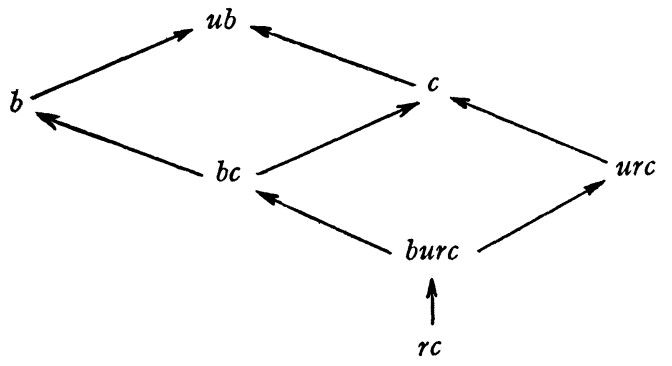

based on a suggestion by him.

The seven types of series under consideration are related as in the diagram, where the arrow indicates implication of the quality at its head by that at its tail.

In $\$ 2$ are listed the conditions to be used in the theorems; $\S 3$ establishes various implications of these conditions; $\$ 4$ is devoted to necessity proofs, and $\$ 5$ to sufficiency proofs. The results of $\S \S 4$ and 5 are tabulated in $\S 6$, it being noted that if $u \subset U$, and $v \subset V$, where $u, U, v, V$ represent classes of series, then $S . U \rightarrow v$ is at once $S .(u, U) \rightarrow(v, V)$ (four cases), and $N . u \rightarrow V$ is at once $N .(u, U) \rightarrow(v, V)$.

Frequent use is made of the following decomposition of double sums, analogous to Abel's partial sum formula for simple series, and due to Hardy.*

$$
\begin{aligned}
\sigma_{m n} \equiv \sum_{k, l=1}^{m, n} x_{k l} b_{k l}= & \sum_{k, l=1}^{m-1, n-1} s_{k l} \Delta_{11} b_{k l} \\
& +\sum_{k=1}^{m-1} s_{k n} \Delta_{10} b_{k n} \\
& +\sum_{l=1}^{n-1} s_{m l} \Delta_{01} b_{m l} \\
& +s_{m n} b_{m n} .
\end{aligned}
$$

Here $s_{m n} \equiv \sum_{k, l=1}^{m, n} x_{k l} ; \Delta_{10} b_{k l} \equiv b_{k l}-b_{k+1, l} ; \Delta_{01} b_{k l} \equiv b_{k l}-b_{k, l+1}$; and $\Delta_{11} b_{k l} \equiv \Delta_{10}\left(\Delta_{01} b_{k l}\right)=\Delta_{01}\left(\Delta_{10} b_{k l}\right)$. The several parts of the decomposition are lettered for future reference.

2. Conditions.

(a) $\sum_{k, l=1}^{\infty}\left|\Delta_{11} b_{k l}\right|=A<\infty$.

* Hardy, On the convergence of certain multiple series, Proceedings of the London Mathematical Society, (2), vol. 1 (1903), pp. 124-128. 

(b) $\quad \sum_{k=1}^{\infty}\left|\Delta_{10} b_{k 1}\right|=B<\infty ; \quad \sum_{l=1}^{\infty}\left|\Delta_{01} b_{1 l}\right|=C<\infty$.
$\lim _{l \rightarrow \infty} \Delta_{10} b_{k l}=0, \quad(k=1,2, \cdots) ;$
$\left(c_{1}\right)$
$\lim _{k \rightarrow \infty} \Delta_{01} b_{k l}=0, \quad(l=1,2, \cdots)$.
(c) $\begin{aligned} \Delta_{10} b_{k l} & =0 \text { for } l>L_{k}, \quad(k=1,2, \cdots) ; \\ \Delta_{01} b_{k l} & =0 \text { for } k>K_{l}, \quad(l=1,2, \cdots) \text {. }\end{aligned}$
(d $\left.\mathrm{d}_{1}\right) \lim _{l \rightarrow \infty} b_{k l}=0,(k=1,2, \cdots) ; \quad \lim _{k \rightarrow \infty} b_{k l}=0,(l=1,2, \cdots)$.
$\left(\mathrm{d}_{2}\right) \begin{aligned} b_{k l} & =0 \text { for } l>J_{k}, \quad(k=1,2, \cdots) ; \\ b_{k l} & =0 \text { for } k>I_{l}, \quad(l=1,2, \cdots) .\end{aligned}$
(e) $\lim _{k, l \rightarrow \infty} b_{k l}=0$.

3. Implications of the Conditions.
(2) ( $\left.\mathrm{c}_{2}\right)$ implies $\left(\mathrm{c}_{1}\right)$.
(3) $\left(\mathrm{d}_{1}\right)$ implies $\left(\mathrm{c}_{1}\right)$.
(4) $\left(\mathrm{d}_{2}\right)$ implies $\left(\mathrm{c}_{2}\right)$.
(5) $\left(\mathrm{d}_{2}\right)$ implies $\left(\mathrm{d}_{1}\right)$.
(6) (a) and (b) imply

$$
\begin{array}{ll}
\sum_{k=1}^{\infty}\left|\Delta_{10} b_{k l}\right| \leqq A+B, & (l=1,2, \cdots) ; \\
\sum_{l=1}^{\infty}\left|\Delta_{01} b_{k l}\right| \leqq A+C, & (k=1,2, \cdots) .
\end{array}
$$

(7) (a) and (b) imply the existence of

$\lim _{l \rightarrow \infty} \Delta_{10} b_{k l}, \quad(k=1,2, \cdots) ; \quad \lim _{k \rightarrow \infty} \Delta_{01} b_{k l}, \quad(l=1,2, \cdots)$.

(8) (a) and (b) imply

$$
\sum_{k=1}^{\infty}\left|\lim _{l \rightarrow \infty} \Delta_{10} b_{k l}\right| \leqq A+B ; \quad \sum_{l=1}^{\infty}\left|\lim _{k \rightarrow \infty} \Delta_{01} b_{k l}\right| \leqq A+C .
$$

(9) (a) and (b) imply that the sequence $\left\{b_{k l}\right\}$ is $r c$.

(10) (a) and ( $\mathrm{c}_{1}$ ) imply (b).

(11) (a) and ( $\mathrm{c}_{1}$ imply

$$
\lim _{l \rightarrow \infty} \sum_{k=1}^{\infty}\left|\Delta_{10} b_{k l}\right|=0 ; \quad \lim _{k \rightarrow \infty} \sum_{l=1}^{\infty}\left|\Delta_{01} b_{k l}\right|=0 .
$$


(12) (a) and ( $\left.\mathrm{c}_{1}\right)$ imply that

$$
\lim _{l \rightarrow \infty} b_{k l}=\lim _{k, l \rightarrow \infty} b_{k l}, \quad(k=1,2, \cdots),
$$

and that

$$
\lim _{k \rightarrow \infty} b_{k l}=\lim _{k, l \rightarrow \infty} b_{k l}, \quad(l=1,2, \cdots) .
$$

(13) (a) and $\left(\mathrm{d}_{1}\right)$ imply (e).

(14) (a), ( $\left.\mathrm{c}_{1}\right)$, and (e) imply $\left(\mathrm{d}_{1}\right)$.

Of these implications, the ones which are not obvious can be proved as indicated below. (Where symmetrical results are indicated, only the first is established.)

Proof of (6). This follows from the identity, for arbitrary $t$,

$$
\sum_{l=1}^{t-1} \Delta_{11} b_{k l}=\Delta_{10} b_{k 1}-\Delta_{10} b_{k t}, \quad(k=1,2, \cdots) .
$$

Proof of (7). By (15),

$$
\lim _{l \rightarrow \infty} \Delta_{10} b_{k l}=\Delta_{10} b_{k 1}-\sum_{l=1}^{\infty} \Delta_{11} b_{k l}, \quad(k=1,2, \cdots) .
$$

Proof of (8). This follows from (16).

Proof of (9). This follows from the identity, for arbitrary $s$ and $t$,

$$
\begin{aligned}
\sum_{k, l=1}^{s-1, t-1} \Delta_{11} b_{k l} & =\sum_{k=1}^{s-1}\left(\Delta_{10} b_{k 1}-\Delta_{10} b_{k t}\right) \\
& =\sum_{k=1}^{s-1} \Delta_{10} b_{k 1}-b_{1 t}+b_{s t}+b_{11}-b_{11} \\
& =\sum_{k=1}^{s-1} \Delta_{10} b_{k 1}+\sum_{l=1}^{t-1} \Delta_{01} b_{1 l}-b_{11}+b_{s t} .
\end{aligned}
$$

Proof of (10). Let $t$ be arbitrary. For any $s$, whatever be $r>t$,

$$
\begin{aligned}
\sum_{k=1}^{s}\left|\Delta_{10} b_{k t}\right| & \leqq \sum_{k=1}^{s}\left|\Delta_{10} b_{k t}-\Delta_{10} b_{k r}\right|+\sum_{k=1}^{s}\left|\Delta_{10} b_{k r}\right| \\
& =\sum_{k=1}^{s}\left|\sum_{l=t}^{r-1} \Delta_{11} b_{k l}\right|+\sum_{k=1}^{s}\left|\Delta_{10} b_{k r}\right| \\
& \leqq \sum_{k=1, l=t}^{\infty}\left|\Delta_{11} b_{k l}\right|+\sum_{k=1}^{s}\left|\Delta_{10} b_{k r}\right|
\end{aligned}
$$


Hence, by $\left(c_{1}\right)$ and the arbitrariness of $s$,

$$
\sum_{k=1}^{\infty}\left|\Delta_{10} b_{k t}\right| \leqq \sum_{k=1, l=t}^{\infty}\left|\Delta_{11} b_{k l}\right| .
$$

Proof of (11). Let $t$ tend to $\infty$ in (17).

Proof of (12). By (10), (9), and $\left(c_{1}\right)$, the sequence $\left\{b_{k l}\right\}$ is $r c$, and

$$
\lim _{l \rightarrow \infty} b_{k l}=\lim _{l \rightarrow \infty} b_{k+1, l}, \quad(k=1,2, \cdots) .
$$

Proof of (13). See (3) and (12).

Proof of (14). See (12).

4. Necessity Proofs. (Only the first parts of symmetrical conditions are dealt with.)

TheOREM 1. N.rc $\rightarrow u b$ are (a) and (b).

The proof for (a) is contained in Theorem 12 of $\mathrm{H}$. That for (b) can be obtained by slight revision of the first argument in Theorem I of K.

Theorem 2. N.urc $\rightarrow u b$ is $\left(\mathrm{c}_{2}\right)$.

By denial of the first part of $\left(c_{2}\right)$, there exists a sequence, for fixed $p$, of non-zero quantities, $\Delta_{10} b_{p l_{i}}$, with $l_{i}>l_{i-1}$. The series with terms

$x_{k l}=\left\{\begin{array}{cl}1 /\left(\Delta_{10} b_{p l_{i}}\right), & \left(k=p, \quad l=l_{i} \text { for } i=1,2, \cdots\right), \\ -1 /\left(\Delta_{10} b_{p l_{i}}\right), & \left(k=p+1, l=l_{i} \text { for } i=1,2, \cdots\right), \\ 0, \text { otherwise }, & \end{array}\right.$

is urc. But, for arbitrary $m>p$ and arbitrary $i$,

$$
\sigma_{m l_{i}}=\sum_{j=1}^{i}\left(\Delta_{10} b_{p l_{j}}\right) /\left(\Delta_{10} b_{p l_{j}}\right)=i .
$$

TheOREM 3. $N . u r c \rightarrow b$ is $\left(\mathrm{d}_{2}\right)$.

By denial of the first part of $\left(d_{2}\right)$, there exists a sequence, for fixed $p$, of non-zero quantities, $b_{p l_{i}}$, with $l_{i}>l_{i-1}$. The series like that defined in Theorem 2, with $\Delta_{10} b_{p l_{i}}$ replaced by $b_{p l_{i}}$, is $u r c$. But for each $i$,

$$
\sigma_{p l_{i}}=\sum_{i=1}^{i}\left(b_{p l_{i}}\right) /\left(b_{p l_{i}}\right)=i
$$


Theorem 4. N.burc $\rightarrow$ c is $\left(\mathrm{c}_{1}\right)$.

Let $p$ be arbitrary. The series with terms

$$
x_{k l}= \begin{cases}(-1)^{l}, & (k=p, \quad l=1,2, \cdots), \\ (-1)^{l+1}, & (k=p+1, l=1,2, \cdots), \\ 0, \text { otherwise }, & \end{cases}
$$

is burc. But, for $m>p$ and arbitrary $n, \sigma_{m n}=\sum_{l=1}^{n}(-1)^{l} \Delta_{10} b_{p l}$, so that $\left|\Delta_{10} b_{p, n+1}\right|=\left|\sigma_{m+1, n+1}-\sigma_{m n}\right|$, which must tend to zero as $m$ and $n$ tend to $\infty$.

Theorem 5. $N . b \rightarrow$ care $\left(\mathrm{d}_{1}\right)$ and $(\mathrm{e})$.

The proof for $\left(\mathrm{d}_{1}\right)$ is given in Theorem II of $\mathrm{K}$; (e) follows from Theorem 1 and (13) above.

Theorem 6. $N . b c \rightarrow$ urc are $\left(\mathrm{d}_{1}\right)$ and $(\mathrm{e})$.

Conditions (a) and $\left(\mathrm{c}_{1}\right)$ are $N . b c \rightarrow u r c$, by Theorems 1 and 4 , respectively. The series with partial sums $s_{k l} \equiv(-1)^{k} / l$, $(k, l=1,2, \cdots)$ is $b c$. In (1), for fixed $n, \lim _{m \rightarrow \infty}(\alpha)$ exists, by (a) $; \lim _{m \rightarrow \infty}(\beta)$ exists, by $(10)$ and $(6) ; \lim _{m \rightarrow \infty}(\gamma)=0$, by $\left(c_{1}\right)$. But by (12) and denial of (e), $\lim _{m \rightarrow \infty}(\delta)$ does not exist. Condition $\left(\mathrm{d}_{1}\right)$ follows from (14).

THEOREM 7. N.burc $\rightarrow r c$ is $\left(\mathrm{d}_{1}\right)$.

Conditions (a) and $\left(\mathrm{c}_{1}\right)$ are $N . b u r c \rightarrow r c$, by Theorems 1 and 4 , respectively. The series with terms

$$
x_{k l}= \begin{cases}(-1)^{k} \quad \text { for } l=1, & (k=1,2, \cdots), \\ (-1)^{k+1} \text { for } l=2, & (k=1,2, \cdots), \\ 0, \text { otherwise }, & \end{cases}
$$

is burc. But $\sigma_{m 1}=\sum_{k=1}^{m}(-1)^{k} b_{k 1}$, so that $\left|b_{m+1,1}\right|=\left|\sigma_{m+1,1}-\sigma_{m 1}\right|$, which must tend to zero as $m$ tends to $\infty$. Condition $\left(\mathrm{d}_{1}\right)$ follows from (12).

5. Sufficiency Proofs. $[(\alpha),(\beta),(\gamma),(\delta)$ are defined in (1). $]$ Theorem 8. $S . b \rightarrow b$ are (a) and (b).

$(\alpha)$ is bounded, by $(\mathrm{a}) ;(\beta)$ and $(\gamma)$ are bounded, by (6); $(\delta)$ is bounded, by (9). 
Theorem 9. S. $u b \rightarrow u b$ are (a) and $\left(\mathrm{c}_{2}\right)$.

Suppose $s_{k l}$ bounded for $k, l>Q$. Set $K \equiv \max K_{l}, \quad(l=1$, $2, \cdots, Q) ; L \equiv \max L_{k},(k=1,2, \cdots, Q) ; R \equiv \max (Q, K, L)$. Since $\Delta_{11} b_{k l}=\Delta_{10}\left(\Delta_{01} b_{k l}\right)=\Delta_{01}\left(\Delta_{10} b_{k l}\right), \quad(\alpha)$ is bounded by $\sum_{k, l=1}^{R}\left|s_{k l} \Delta_{11} b_{k l}\right|+\sum_{k, l=Q+1}^{\infty}\left|s_{k l} \Delta_{11} b_{k l}\right|$. For $n>R,(\beta)$ reduces to $\sum_{k=Q+1}^{m-1} s_{k n} \Delta_{10} b_{k n}$, which is bounded, by (2), (10), and (6). Similarly, for $m>R,(\gamma)$ is bounded. For $m, n>R,(\delta)$ is bounded by (2), (10), and (9).

TheOREm 10. S.ub $\rightarrow$ rc are (a) and $\left(\mathrm{d}_{2}\right)$.

The conditions are $S . u b \rightarrow c$, by (4), (5), (13), and Theorem 15 (below). For fixed $n, \sigma_{m n}=\sum_{l=1, k=1}^{n, I_{l}} x_{k l} b_{k l}$ for all $m>\max I_{l}$, $(l=1,2, \cdots, n)$. Similarly, $\lim _{n \rightarrow \infty} \sigma_{m n}$ exists for $m=1,2, \cdots$.

Theorem 11. S.rc $\rightarrow$ rc are (a) and (b). See Theorem 10 of H.

TheOREM 12. S.bc $\rightarrow$ bc are (a) and $\left(\mathrm{c}_{1}\right)$.

By (10) and Theorem 8, the conditions are $S . b c \rightarrow b$. Let $m$, $n$ tend to $\infty$. $(\alpha)$ converges, by $(\mathrm{a}) ;(\beta)$ and $(\gamma)$ tend to zero, by $(11) ;(\delta)$ converges, by $(10)$ and $(9)$.

Theorem 13. S.b $\rightarrow$ rc are $(\mathrm{a})$ and $\left(\mathrm{d}_{1}\right)$.

Use (3), (10), and (13); and see Theorem 11 of $\mathrm{H}$.

TheOREM 14. S.c $\rightarrow$ c are (a) and $\left(\mathrm{c}_{2}\right)$.

Suppose $s_{k l}$ bounded for $k, l>Q$. Define $K, L, R$, as in Theorem 9 . As $m, n$ tend to $\infty,(\alpha)$ converges to

$$
\sum_{k, l=1}^{R} s_{k l} \Delta_{11} b_{k l}+\sum_{k, l=Q+1}^{\infty} s_{k l} \Delta_{11} b_{k l}-\sum_{k, l=Q+1}^{R} s_{k l} \Delta_{11} b_{k l} .
$$

For $n>R,(\beta)$ reduces to $\sum_{k=Q+1}^{m-1} s_{k n} \Delta_{10} b_{k n}$, which converges to zero, by (2) and (11). Similarly, it can be shown that $(\gamma)$ tends to zero. The sum $(\delta)$ converges, by (2), (10), and (9). (Compare the concluding argument in Theorem I of $\mathrm{K}$.)

Theorem 15. S.ub $\rightarrow$ urc are $(\mathrm{a}),\left(\mathrm{c}_{2}\right)$, and (e).

Suppose $s_{k l}$ bounded for $k, l>Q$. Define $K, L, R$, again as in Theorem 9. As $m$ and $n$ tend to $\infty,(\alpha),(\beta)$, and $(\gamma)$ converge, as is seen by reasoning as in Theorem $14 ;(\delta)$ tends to zero, by (e). 
Now fix $n>R$. Then for all $m>\max K_{l},(l=1,2, \cdots, n-1)$, $(\alpha)$ becomes $\sum_{l=1, k=1}^{n-1, K_{l}} s_{k l} \Delta_{11} b_{k l} ;(\beta)$ becomes $\sum_{k=Q+1}^{m-1} s_{k n} \Delta_{10} b_{k n}$, and as $m$ tends to $\infty$ this expression converges, by (2), (10), and $(6) ;(\gamma)$ converges to zero, by $(2) ;(\delta)$ tends to zero, by $(2)$ and (14). Similarly, it can be shown that $\lim _{n \rightarrow \infty} \sigma_{m n}$ exists for each $m>R$.

TheOREM 16. S.burc $\rightarrow$ burc are (a) and $\left(\mathrm{c}_{1}\right)$.

The conditions are $S . b u r c \rightarrow b c$ by Theorem 12. Suppose $s_{k l}$ regularly convergent for $k, l>Q$. For fixed $n>Q$, as $m$ tends to infinity, $(\alpha)$ converges, by $(\mathrm{a}) ;(\beta)$ converges, by (10) and (6); $(\gamma)$ converges to zero, by $\left(c_{1}\right) ;(\delta)$ converges, by $(10)$ and $(9)$. Similarly, it can be shown that for fixed $m>Q, \lim _{n \rightarrow \infty} \sigma_{m n}$ exists.

Theorem 17. S.urc $\rightarrow$ urc are (a) and $\left(\mathrm{c}_{2}\right)$.

The conditions are $S . u r c \rightarrow c$, by Theorem 14. Suppose $s_{k l}$ regularly convergent for $k, l>Q$. Define $K, L, R$, in a manner analogous to that used in Theorem 9 . If $n>R$, it can be shown as in Theorem 15 that, as $m \rightarrow \infty,(\alpha),(\beta),(\gamma)$ converge. $(\delta)$ converges, by (2), (10), and (9).

6. Summary of Results.

Transformation

$$
\begin{aligned}
r c \rightarrow & \rightarrow u b \\
b u r c \rightarrow & u b \\
b c \rightarrow & u b \\
b \rightarrow & u b \\
u r c \rightarrow & \rightarrow b b \\
c \rightarrow & u b \\
u b \rightarrow & u^{\prime} \\
r c \rightarrow & b \\
b u r c \rightarrow & b \\
b c \rightarrow & b \\
b \rightarrow & b \\
u r c \rightarrow & b \\
c \rightarrow & b \\
u b \rightarrow & b
\end{aligned}
$$

N. Conditions

(a), (b)

(a), (b)

(a), (b)

(a), (b)

(a), (b), $\left(\mathrm{c}_{1}\right),\left(\mathrm{c}_{2}\right)$

(a), (b), (c,,$\left(c_{2}\right)$

(a), (b), $\overrightarrow{\left(c_{1}\right)},\left(c_{2}\right)$

(a), (b)

(a), (b)

(a), (b)

(a), (b)

(a), (b), $\left(\mathrm{c}_{1}\right),\left(\mathrm{c}_{2}\right),\left(\mathrm{d}_{1}\right),\left(\mathrm{d}_{2}\right),(\mathrm{e})$

(a), (b), $\overline{\left(c_{1}\right)},\left(c_{2}\right), \overline{\left(d_{1}\right),},\left(d_{2}\right), \overline{(e)}$

(a), (b), $\overline{\left(c_{1}\right)},\left(c_{2}\right), \overline{\left(d_{1}\right),}\left(d_{2}\right), \overline{(e)}$
S. Conditions
(a), (b)
(a), (b)
(a), (b)
(a), (b)
(a), $\left(\mathrm{c}_{2}\right)$
(a), $\left(\mathrm{c}_{2}\right)$
(a), $\left(\mathrm{c}_{2}\right)$

(a), (b)

(a), (b)

(a), (b)

(a), (b)

(a), $\left(\mathrm{d}_{2}\right)$

(a), $\left(\mathrm{d}_{2}\right)$

(a), $\left(\mathrm{d}_{2}\right)$ 
Transformation

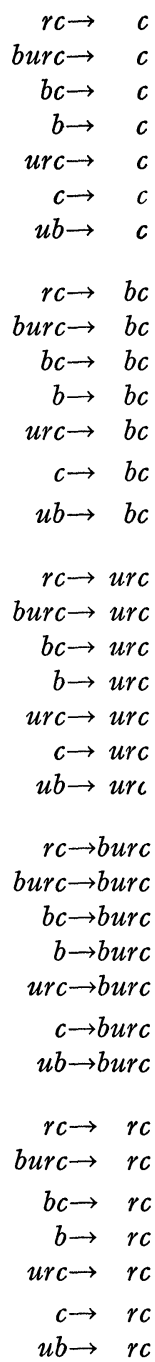

N. Conditions

(a), (b)

(a), (b), (c $c_{1}$

(a), (b), (c $\left.c_{1}\right)$

(a), (b), $\left(c_{1}\right),\left(d_{1}\right),(e)$

(a), (b), $\left(c_{1}\right),\left(c_{2}\right)$

(a), (b), (c $\left.c_{1}\right),\left(c_{2}\right)$

(a), (b), (c $\left.c_{1}\right),\left(c_{2}\right),\left(d_{1}\right),(e)$

(a), (b)

(a), (b), (c $\left.c_{1}\right)$

(a), (b), (c $\left.c_{1}\right)$

(a), (b), $\left(c_{1}\right),\left(d_{1}\right),(e)$

(a), (b), $\left(\mathrm{c}_{1}\right),\left(\mathrm{c}_{2}\right),\left(\mathrm{d}_{1}\right),\left(\mathrm{d}_{2}\right),(\mathrm{e})$

(a), (b), (c $\left.c_{1}\right),\left(c_{2}\right), \overline{\left(d_{1}\right),}\left(d_{2}\right), \overline{(e)}$

(a), (b), $\left(c_{1}\right),\left(c_{2}\right), \overline{\left(d_{1}\right),}\left(d_{2}\right), \overline{(e)}$

(a), (b)

(a), (b), (c 1 )

(a), (b), $\left(c_{1}\right),\left(d_{1}\right),(e)$

(a), (b), $\left(c_{1}\right),\left(d_{1}\right),(e)$

(a), (b), (c $\left.c_{1}\right),\left(c_{2}\right)$

(a), (b), (c $\left.c_{1}\right),\left(c_{2}\right),\left(d_{1}\right),(e)$

(a), (b), $\left(c_{1}\right),\left(c_{2}\right),\left(d_{1}\right),(e)$

\section{(a), (b)}

(a), (b), ( $\left.c_{1}\right)$

(a), (b), $\left(c_{1}\right),\left(d_{1}\right),(e)$

(a), (b), $\left(c_{1}\right),\left(d_{1}\right),(e)$

(a), (b), $\left(\mathrm{c}_{1}\right),\left(\mathrm{c}_{2}\right),\left(\mathrm{d}_{1}\right),\left(\mathrm{d}_{2}\right),(\mathrm{e})$

(a), (b), (c $\left.\mathrm{c}_{1}\right),\left(\mathrm{c}_{2}\right), \overrightarrow{\left(\mathrm{d}_{1}\right)},\left(\mathrm{d}_{2}\right), \overrightarrow{\mathrm{e}}$

(a), (b), $\left(c_{1}\right),\left(c_{2}\right),\left(d_{1}\right),\left(d_{2}\right),(e)$

(a), (b)

(a), (b), $\left(c_{1}\right),\left(d_{1}\right),(e)$

(a), (b), $\left(c_{1}\right),\left(d_{1}\right), \overline{(e)}$

(a), (b), $\left(\mathrm{c}_{1}\right),\left(\mathrm{d}_{1}\right),(\mathrm{e})$

(a), (b), $\left(c_{1}\right),\left(c_{2}\right),\left(d_{1}\right),\left(d_{2}\right),(e)$

(a), (b), (c $\left.c_{1}\right),\left(c_{2}\right),\left(d_{1}\right),\left(d_{2}\right), \overline{(e)}$

(a), (b), (c $\left.c_{1}\right),\left(c_{2}\right),\left(d_{1}\right),\left(d_{2}\right),(e)$
S. Conditions

(a), (b)

(a), $\left(c_{1}\right)$

(a), $\left(c_{1}\right)$

(a), $\left(\mathrm{d}_{1}\right)$

(a), $\left(\mathrm{c}_{2}\right)$

(a), $\left(c_{2}\right)$

(a), $\left(c_{2}\right),(e)$

(a), (b)

(a), $\left(c_{1}\right)$

(a), $\left(c_{1}\right)$

(a), $\left(\mathrm{d}_{1}\right)$

(a), $\left(\mathrm{d}_{2}\right)$

(a), $\left(\mathrm{d}_{2}\right)$

(a), $\left(\mathrm{d}_{2}\right)$

(a), (b)

(a), $\left(c_{1}\right)$

(a), $\left(\mathrm{d}_{1}\right)$

(a), $\left(d_{1}\right)$

(a), $\left(c_{2}\right)$

(a), $\left(\mathrm{c}_{2}\right),(\mathrm{e})$

(a), $\left(\mathrm{c}_{2}\right),(\mathrm{e})$

(a), (b)

(a), $\left(c_{1}\right)$

(a), $\left(\mathrm{d}_{1}\right)$

(a), $\left(\mathrm{d}_{1}\right)$

(a), $\left(\mathrm{d}_{2}\right)$

(a), $\left(\mathrm{d}_{2}\right)$

(a), $\left(\mathrm{d}_{2}\right)$

(a), (b)

(a), $\left(\mathrm{d}_{1}\right)$

(a), $\left(\mathrm{d}_{1}\right)$

(a), $\left(\mathrm{d}_{1}\right)$

(a), $\left(\mathrm{d}_{2}\right)$

(a), $\left(\mathrm{d}_{2}\right)$

(a), $\left(\mathrm{d}_{2}\right)$

Those entries which do not follow from Theorems 1-17 can be deduced from accompanying entries by application of (2), (5), or (13). Such entries are underscored.

\section{BROWN UNIVERSITY}

